\title{
Mechanisms of Berberine (Natural Yellow 18)-Induced Mitochondrial Dysfunction: Interaction with the Adenine Nucleotide Translocator
}

\author{
Cláudia V. Pereira, Nuno G. Machado, and Paulo J. Oliveira ${ }^{1}$ \\ Center of Neurosciences and Cell Biology, Department of Zoology, University of Coimbra, 3004-517 Coimbra, Portugal
}

Received May 13, 2008; accepted June 26, 2008

\begin{abstract}
Berberine [Natural Yellow 18, 5,6-dihydro-9,10-dimethoxybenzo(g)-1,3-benzodioxolo (5,6- $a$ ) quinolizinium] is an alkaloid present in plants of the Berberidaceae family and used in traditional Chinese and North American medicine. We have previously demonstrated that berberine causes mitochondrial depolarization and fragmentation, with simultaneous increase in oxidative stress. We also demonstrated that berberine causes an inhibition of mitochondrial respiration and a decrease on calcium loading capacity through induction of the mitochondrial permeability transition (MPT). The objective of the present work is to investigate a common target for both induction of the MPT and inhibition of respiration. The hypothesis is that berberine induces the MPT through interacting with the adenine nucleotide translocator (ANT). By measuring induction of the MPT through increased mitochondrial swelling, membrane depolarization and loss of calcium retention, we observed that the effects of berberine were not inhibited by bongkrekic acid although adenosine diphosphate (ADP)/oligomycin completely prevented the MPT. Also, we observed that berberine increased the depolarization effect of oleic acid on liver mitochondria. The initial depolarization observed when berberine is added to mitochondria was not affected by ANT inhibitors. Taken together, we propose that berberine acts on the ANT, altering the binding of the protein to bongkrekic acid but not to cyclosporin A or ADP. It is also clear that the membrane potential is required for berberine effects, most likely for allowing for its mitochondrial accumulation. Mitochondrial effects of berberine can be relevant not only for its proposed antitumor activity but also for the assessment of its organ toxicity, depending on factors such as tissue accumulation or delivery.
\end{abstract}

Key Words: Berberine; toxicology; transition pore; mitochondria; adenine nucleotide translocator.

Berberine [Natural Yellow 18, 5,6-dihydro-9,10-dimethoxybenzo(g)-1,3-benzodioxolo (5,6- $a$ ) quinolizinium], a benzyl tetra isoquinoline plant alkaloid derived from the Berberidaceae family, has been extensively used for many centuries. In fact, extracts containing berberine have been used in the traditional Chinese and Native American medicine. Berberine

\footnotetext{
${ }^{1}$ To whom correspondence should be addressed at Center of Neurosciences and Cell Biology, Department of Zoology, University of Coimbra, 3004-517 Coimbra, Portugal. Fax: +351-239855789. E-mail: pauloliv@ci.uc.pt.
}

chemical structure (Fig. 1) contains a quaternary base and it is commercially available as various salts such as berberine chloride and hemisulfate. Many pharmacological applications have been attributed to berberine, such as antidiarrheic (Taylor and Baird, 1995), antimicrobial (Kaneda et al., 1991), antiinflammatory (Ckless et al., 1995), antiarrhythmic (Hong et al., 2002), antihypertensive (Hong et al., 2002), antiproliferative/ antitumoral (Letasiova et al., 2005; Serafim et al., 2008), and as an antioxidant (Shirwaikar et al., 2006). It has also been reported that berberine reduces glucose blood levels in diabetes (Zhou et al., 2007), alters the processing of Alzheimer's amyloid precursor protein decreasing $\mathrm{A} \beta$ secretion (Asai et al., 2007), and shows antiangiogenic properties (Lin et al., 2004), inhibiting HIF- $\alpha$ expression via enhanced proteolysis. Berberine is metabolized in the liver by cytochrome P450, suffering phase I metabolism (Pereira et al., 2007)

Previously, we demonstrated that berberine is selectively accumulated by mitochondria on K1735-M2 melanoma cells, arresting cell proliferation, causing mitochondrial fragmentation and depolarization, oxidative stress and a decrease in ATP levels (Pereira et al., 2007). A decrease in the number of mitochondria-like structures and in mitochondrial DNA copy number was also found (Pereira et al., 2007). The work by Barreto et al. (2003) also confirmed the relevance of positively charges on the selective accumulation of alkaloids in mitochondria. We have identified two distinct types of effects of berberine on isolated mitochondrial fractions. Berberine inhibits state 3 and uncoupled respiration, which is more visible when complex I substrates are used (Pereira et al., 2007). On the other hand, berberine also decreases mitochondrial calcium loading capacity through enhanced induction of the mitochondrial permeability transition (MPT) (Pereira et al., 2007). Through the analyses of several parameters, it is proposed that berberine inhibits the phosphorylative system, although no effects on the ATPase activity were observed (Pereira et al., 2007). The interaction of berberine on both the phosphorylative system and on the MPT suggests that a common target may be involved. One interesting candidate is the adenine nucleotide translocator (ANT).

The ANT is a mitochondrial inner membrane adenosine diphosphate (ADP)-ATP antiporter that imports ADP to the 


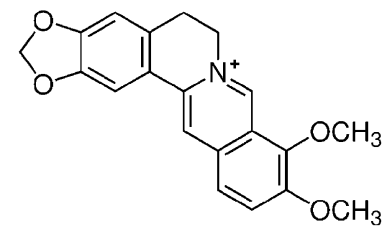

FIG. 1. Chemical structure of berberine.

matrix and exports ATP to the cytosol, being suggested to be a structural component of the MPT pore (Armstrong, 2006), although recent data cast doubts regarding this issue (Juhaszova et al., 2008). The ANT alternates between two distinct conformations in which adenine nucleotides are either bound to the cytosolic side (c-state) or to the matrix side (m-state) of the inner mitochondrial membrane. It has also been described that the c-conformation is favorable to the MPT (Armstrong, 2006). Carboxyatractyloside which binds to ANT in the c-state activates the MPT pore, whereas bongkrekic acid which binds to ANT in the m-state inhibits the MPT pore. The two ligands are known to be ANT specific inhibitors (Armstrong, 2006).

The role of the ANT in the MPT has not been fully resolved. Although the MPT is an inner membrane phenomenon, there is substantial evidence of the involvement of the VDAC (voltagedependent anion channel) in the MPT. Crompton et al. (1998) showed that matrix cyclophilin D (CycP-D), the third putative component of the MPT, binds to complexes of VDAC and ANT in order to form the MPT complex. It has also been reported that CycP-D is more of a regulatory component of the MPT pore than a structural one. Cyclosporin A (Cyc A) was show to block the MPT by inhibiting the binding of CycP-D to the ANT (Szabo and Zoratti, 1991).

MPT activation compromises the normal integrity of the mitochondrial inner membrane resulting into uncoupled oxidative phosphorylation, ATP decay, mitochondrial swelling and release of apoptogenic factors, indicating a key role for the MPT in apoptosis, although this subject is still controversial (Armstrong, 2006).

In the present work, we proposed to investigate the mechanism by which berberine induced the MPT pore in isolated rat liver mitochondria; we hypothesize that berberine induces that phenomenon by interacting with the ANT. Experiments with specific ANT ligands (bongkrekic acid, carboxyatractyloside) were performed in order to identify the ANT as the site for berberine action on isolated liver mitochondria.

\section{MATERIALS AND METHODS}

Materials. Berberine hemisulfate was obtained from Sigma-Aldrich (St Louis, MO) and prepared in dimethyl sulfoxide (DMSO), which is routinely used as a solvent for isolated mitochondrial studies. The total volume of DMSO was always smaller than $0.1 \%$, which had negligible effects in all experiments.
Concentrations used in this study were based in previous literature that showed effects in both intact melanoma cells and isolated hepatic mitochondria (Pereira et al., 2007).

Calcium Green-5N, hexapotassium salt, was obtained from Invitrogen (Carlsbad, CA) and Bongkrekic acid was obtained from Calbiochem (San Diego, CA, purity: $\geq 92 \%$ by high-performance liquid chromatography). Carboxyatractyloside was obtained from Sigma-Aldrich.

All other compounds were of the highest grade of purity commercially available.

Animals. Male Wistar rats (6-10 weeks old), housed at $22 \pm 2^{\circ} \mathrm{C}$ under artificial light for a 12-h light/day cycle and with free access to water and food, were used throughout the experiments. The research procedure was carried out in accordance with the European Requirement for Vertebrate Animal Research and within the internal Standards for Animal Research at the CNC.

Isolation of rat liver mitochondria. Mitochondria were isolated from livers of male Wistar rats by conventional methods with slight modifications (Pereira $e t$ al., 2007). Homogenization medium contained $250 \mathrm{mM}$ sucrose, $10 \mathrm{mM} 4-(2-$ Hydroxyethyl)piperazine-1-ethanesulfonic acid (HEPES), pH 7.4, 1mM EGTA, and $0.1 \%$ fat-free bovine serum albumin (BSA). Ethylene-bis(oxyethylenenitrilo)tetraacetic acid (EGTA) and BSA were not present at the final washing medium, adjusted at $\mathrm{pH}$ 7.4. The mitochondrial pellet was washed twice, suspended in the washing medium, and immediately used. Protein content was determined by the biuret method (Gornall et al., 1949) calibrated with BSA.

Mitochondrial swelling. Mitochondrial volume changes were followed by the decrease of absorbance at $540 \mathrm{~nm}$ with a Jasco V-560 spectrophotometer (Jasco, Tokyo, Japan). The assays were performed in $2 \mathrm{ml}$ of the reaction medium $(200 \mathrm{mM}$ sucrose, $10 \mathrm{mM}$ Tris- $\mathrm{HCl}, \mathrm{pH} 7.4,10 \mu \mathrm{M}$ EGTA, $1 \mathrm{mM}$ $\left.\mathrm{KH}_{2} \mathrm{PO}_{4}\right), 0.6 \mu \mathrm{M}$ rotenone plus $5 \mathrm{mM}$ succinate for complex II-related assays or $5 \mathrm{mM}$ glutamate/malate for complex I assays to which $1.5 \mathrm{mg}$ of mitochondrial protein was added under constant stirring at $30^{\circ} \mathrm{C}$.

Calcium (26.7-53.3 nmol/mg protein) was added to the preparation approximately $1 \mathrm{~min}$ after the start of the experiment. Cyc A $(1 \mu \mathrm{M})$ was added to the mitochondrial preparation before the addition of calcium to inhibit the MPT. Berberine was preincubated with the mitochondrial preparation for approximately $1-2 \mathrm{~min}$.

The ANT inhibitors carboxyatractyloside $(2.5 \mu \mathrm{M})$ and bongkrekic acid $(12.5 \mu \mathrm{M})$ were also used in some experiments in order to modulate the effect of berberine on the MPT. The concentrations of the two ANT inhibitors were chosen with basis in preliminary experiments, regarding inhibition of ADP phosphorylation and MPT modulation (data not shown). Both carboxyatractyloside and bongkrekic acid were added to the mitochondrial preparation before calcium addition. Different concentrations of berberine were preincubated for $1 \mathrm{~min}$ with the mitochondrial preparation.

Mitochondrial calcium loading. Extramitochondrial free $\mathrm{Ca}^{2+}$ was assayed by using the hexapotassium salt of the fluorescent probe Calcium Green 5-N $(1 \mu \mathrm{M})$.

Liver mitochondria $(0.75 \mathrm{mg})$ were suspended in $2 \mathrm{ml}$ of buffer containing $200 \mathrm{mM}$ sucrose, $10 \mathrm{mM}$ Tris, $10 \mu \mathrm{M}$ EGTA, and $1 \mathrm{mM} \mathrm{KH}_{2} \mathrm{PO}_{4}$. Rotenone $(0.6 \mu \mathrm{M})$ and succinate $(5 \mathrm{mM})$ were also added to the media.

Fluorescence was continuously recorded in a water-jacketed cuvette holder at $30^{\circ} \mathrm{C}$ using a PerkinElmer LS-55 fluorescence spectrometer (PerkinElmer Life and Analytical Sciences, Boston, MA) with excitation and emission wavelengths of 506 and 531, respectively. Slits used were $5 \mathrm{~nm}$ for both excitation and emission.

Different concentrations of berberine were added to the mitochondrial preparation $(0.75 \mathrm{mg})$, before the addition of calcium $(10-13.3 \mathrm{nmol} / \mathrm{mg}$ protein) which was made 1 min after the start of the experiment.

Cyc A $(1 \mu \mathrm{M})$ was added to the mitochondrial preparation before the addition of calcium to inhibit the MPT. In some assays, carboxyatractyloside $(2.5 \mu \mathrm{M})$ and bongkrekic acid $(12.5 \mu \mathrm{M})$ were added before calcium addition.

Measurement of the mitochondrial transmembrane potential. The mitochondrial transmembrane potential $(\Delta \psi)$ was indirectly estimated by the 
mitochondrial accumulation of the lipophilic cation-tetraphenylphosphonium cation $\left(\mathrm{TPP}^{+}\right)$as detected by using a $\mathrm{TPP}^{+}$selective electrode in combination with an $\mathrm{Ag} / \mathrm{AgCl}$ saturated reference electrode. Both the $\mathrm{TPP}^{+}$electrode and the reference electrode were inserted into an open vessel with magnetic stirring and were connected to a pH meter (model 3305, Jenway, Essex, UK). The signals were fed into a potentiometric recorder (model BD 121,Kipp \& Zonen B.V., Delft, The Netherlands). No correction was made for "passive" binding of $\mathrm{TPP}^{+}$to the mitochondrial membranes because the purpose of the experiments was to show relative changes in potentials rather than absolute values.

Mitochondrial protein $(1.5 \mathrm{mg})$ was suspended under constant stirring in $1 \mathrm{ml}$ of reaction medium composed $125 \mathrm{mM}$ sucrose, $65 \mathrm{mM} \mathrm{KCl}, 5 \mathrm{mM}$ $\mathrm{KH}_{2} \mathrm{PO}_{4}, 2.5 \mathrm{mM} \mathrm{MgCl}$, and $5 \mathrm{mM}$ HEPES, $\mathrm{pH} 7.4\left(30^{\circ} \mathrm{C}\right)$, supplemented with $3 \mu \mathrm{M} \mathrm{TPP}{ }^{+}$. Mitochondria were energized with $5 \mathrm{mM}$ of succinate plus $3 \mu \mathrm{M}$ rotenone.

Different concentrations of berberine were added before mitochondrial protein. Forty to $53.3 \mathrm{nmol}$ of calcium/mg protein was added to initiate the MPT.

Absolute values for membrane potential (in $\mathrm{mV}$ ) were determined from the equation originally proposed by (Kamo et al., 1979), assuming Nernst distribution of the ion across the membrane electrode.

Cyc A $(1 \mu \mathrm{M})$ was added to the mitochondrial preparation before the addition of calcium to inhibit the MPT. As described before, carboxyatractyloside $(2.5 \mu \mathrm{M})$, bongkrekic acid $(12.5 \mu \mathrm{M})$, or ADP $(500 \mu \mathrm{M})$ plus oligomycin $(20 \mu \mathrm{g})$ were also preincubated with mitochondria.

In order to investigate the effects of berberine on oleic acid-induced depolarization, freshly isolated mitochondria $(1.5 \mathrm{mg})$ were energized using $5 \mathrm{mM}$ succinate plus $3 \mu \mathrm{M}$ rotenone and were allowed to stabilize at the maximum membrane potential for $60 \mathrm{~s}$.
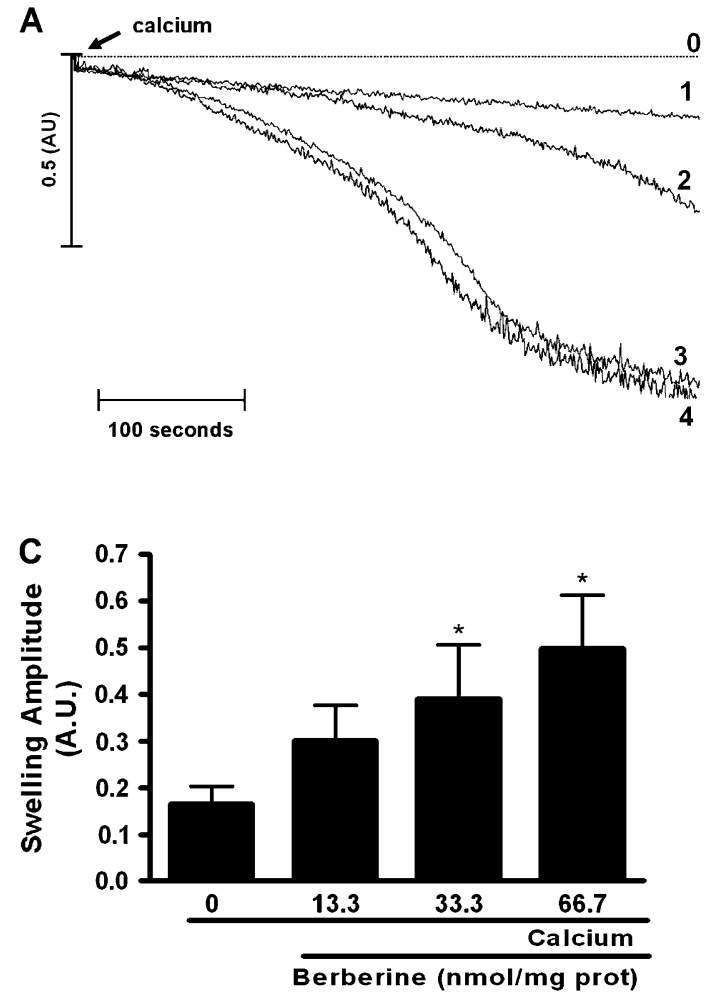

After $\Delta \Psi$ stabilization, $0.84 \mathrm{nmol} / \mathrm{mg}$ (four additions total $=6.88 \mathrm{nmol} / \mathrm{mg}$ protein) of oleic acid was added to induce membrane depolarization. Different concentrations of berberine were also added before initiating the assay.

In selected experiments, carboxyatractyloside $(2.5 \mu \mathrm{M})$ or carbonyl cyanide 4-(trifluoromethoxy)phenylhydrazone (FCCP) $(0.1 \mu \mathrm{M})$ was added to the mitochondrial fractions.

Statistical analysis. Data are expressed as means \pm SEM and evaluated by one-away ANOVA followed by Bonferroni multiple comparison tests.

Differences were considered significant if the $p<0.05$.

\section{RESULTS}

\section{Berberine Induces the MPT in Isolated Hepatic \\ Mitochondrial Fractions}

Calcium-dependent mitochondrial swelling measured by light scattering at $540 \mathrm{~nm}$ is a characteristic measure of MPT induction (Hunter and Haworth, 1979). In a first approach we investigated whether berberine was able to induce the MPT with complex I substrates, in a similar fashion to what was previously observed for complex II substrates (Pereira et al., 2007). Figures 2A-D demonstrate that berberine in a dosedependent fashion was able to induce mitochondrial swelling upon calcium addition, irrespective of the substrate used.
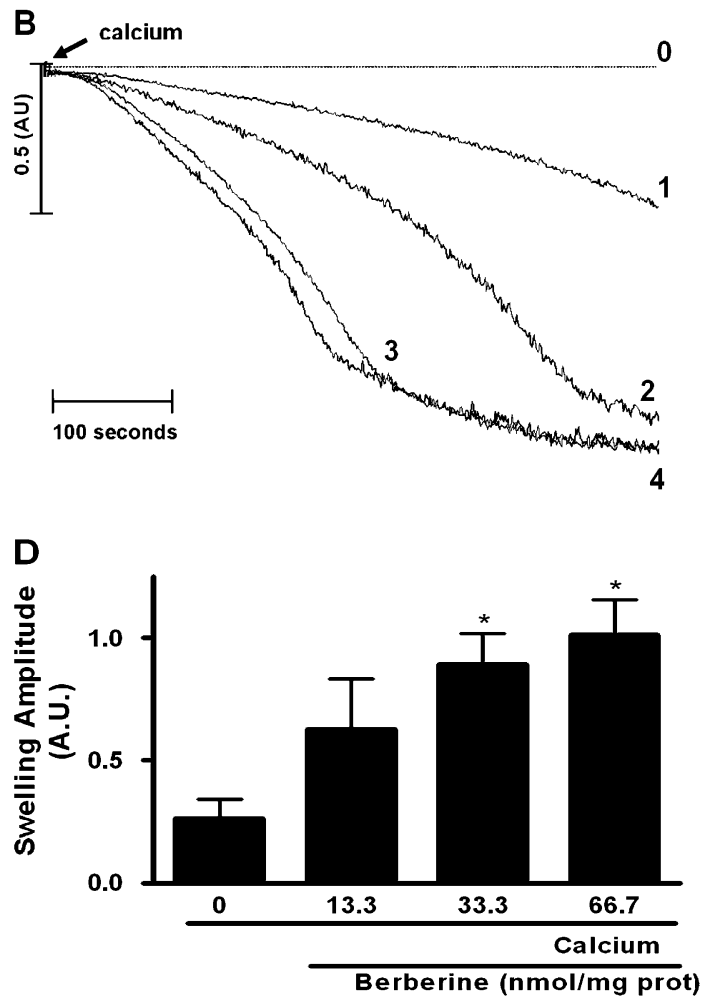

FIG. 2. Mitochondria were incubated with different concentrations of 13.3, 33.3 and $66.7 \mathrm{nmol}$ berberine/mg protein and with succinate plus rotenone or glutamate/malate as substrates. Calcium (26.7-53.3 nmol/mg protein) was added to the preparation in order to induce the MPT pore. (A and C) Typical recording and amplitude of swelling recorded $400 \mathrm{~s}$ after calcium addition, when glutamate-malate was used as substrate. (B and D) Typical recording and amplitude of swelling recorded $400 \mathrm{~s}$ after calcium addition, when succinate was used as substrate. (C and D) Bars represent the mean \pm SEM of 6/7 different preparations. $(*$ ) Statistically different compared with the control $(p<0.05$ ). (A and B) 0 , reference line; 1 , control; $2,13.3 \mathrm{nmol}$ berberine/mg protein; 3 , $33.3 \mathrm{nmol}$ berberine/mg protein; 4, $66.7 \mathrm{nmol}$ berberine/mg protein. 
Succinate was then used as the substrate for the following experiments regarding MPT induction.

We followed up the selecting one berberine concentration (133.3 nmol/mg protein) and investigating whether classic ANT inhibitors such as carboxyatractyloside and bongkrekic acid could interfere with the action of berberine on the MPT (Fig. 3). Bongkrekic acid was previously shown to inhibit MPT induction in isolated hepatic (Vergun and Reynolds, 2005) and cardiac mitochondria (Haworth and Hunter, 2000).

As expected, in the absence of berberine, bongkrekic acid was able to inhibit the MPT, as opposed to carboxyatractyloside which increased calcium-induced mitochondrial swelling. The most relevant result was that bongkrekic acid was not able to inhibit berberine-induced mitochondrial swelling (Fig. 3B). The addition of $\mathrm{Cyc} A$, the classic MPT pore inhibitor (Broekemeier et al., 1989), to mitochondria incubated with
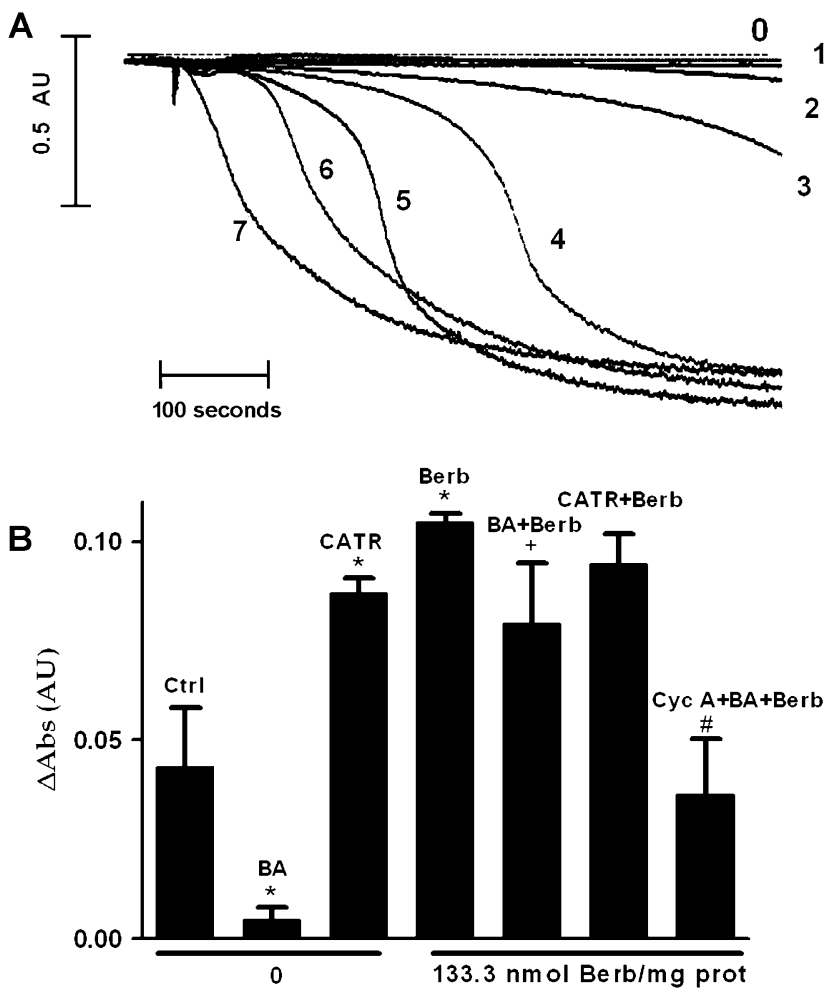

FIG. 3. Effects of ANT inhibitors on berberine-induced swelling. (A) Typical recording. Mitochondria were preincubated with the presence and absence of $133.3 \mathrm{nmol}$ berberine/mg protein and/or carboxyatractyloside (CATR) and bongkrekic acid (BA); Calcium (26.7-53.3 nmol/mg protein) was then added to the preparation in order to induce the MPT pore. Mitochondrial swelling traces are representative of six repetitions, each using different mitochondrial preparations from separate animals. Legend: 0- Reference line; 1, bongkrekic acid (BA); 2, Cyc A; 3, control (Ctrl); 4, CATR; 5, berberine (berb); 6, BA + berb; 7, CATR + berb. (B) Data represent the amplitude of absorbance measured before and $400 \mathrm{~s}$ after calcium addition. Bars represent the mean \pm SEM of six repetitions each analyzed by one-way ANOVA. Differences were considered significant if $p<0.05$. *, Ctrl versus BA, CATR, and berb; +, BA versus BA + berb; \#, BA + berb versus Cyc A + BA + berb. bongkrekic acid plus berberine was able to decrease mitochondrial swelling.

In order to verify if berberine effects on MPT were dependent on the mitochondrial transmembrane potential, we performed swelling assays with nonenergized mitochondria, where mitochondrial calcium loading was obtained through a calcium ionophore.

The results described in Figure 4 demonstrate that in the absence of a transmembrane electric potential, berberine does not cause mitochondrial swelling in the presence of calcium.

\section{Berberine Induces Mitochondrial Depolarization Dependent on Calcium}

Calcium-induced MPT opening leads to the loss of $\Delta \Psi$ in a Cyc A-sensitive fashion (Pereira et al., 2007). Figure 5 illustrates a typical experimental of measuring the effect of different concentrations of berberine $(6.7,13.3,33.3$, and $66.7 \mathrm{nmol} / \mathrm{mg}$ protein) on calcium-induced mitochondrial depolarization.

Mitochondria were preincubated with different concentrations of berberine for $60 \mathrm{~s}$ after being energized with succinate. Figure 5 shows typical recordings on the effect of berberine on calcium-induced depolarization. A graphic pictured in Figure $5 B$ describes the values of $\Delta \Psi$ before, 5 and $10 \mathrm{~min}$ after calcium addition.

The results show that $13.3,33.3$, and $66.7 \mathrm{nmol}$ berberine/ mg protein significantly cause mitochondrial depolarization upon calcium addition. Cyc A was able to prevent the loss of $\Delta \Psi$ in the presence of calcium. Confirming results obtained with mitochondrial swelling, it was observed that the effect of carboxyatractyloside is increased by the presence of berberine (i.e., increasing calcium-induced depolarization) and that the inhibitory effect of bongkrekic acid on the loss of $\Delta \Psi$ is not

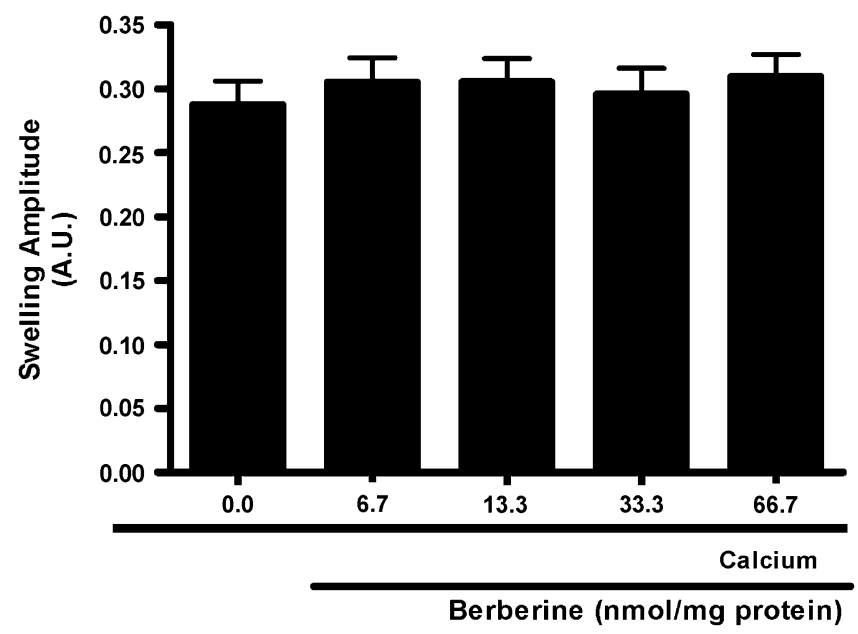

FIG. 4. Berberine does not cause mitochondrial swelling in nonenergized mitochondria. Data represent the swelling amplitude before and $400 \mathrm{~s}$ after calcium addition. Bars represent the mean \pm SEM of three separate experiments. 
observable in the presence of berberine (Fig. 6). Cyc A was able to completely inhibit calcium-induced depolarization in the presence of berberine and bongkrekic acid.

\section{Berberine Decreases Mitochondrial Calcium Loading Capacity}

To obtain further evidence that berberine induces MPT pore opening, mitochondrial calcium loading capacity in the presence of different concentrations of the molecule was evaluated. Increased MPT induction translates into a decrease ability of mitochondria to retain calcium. Extramitochondrial
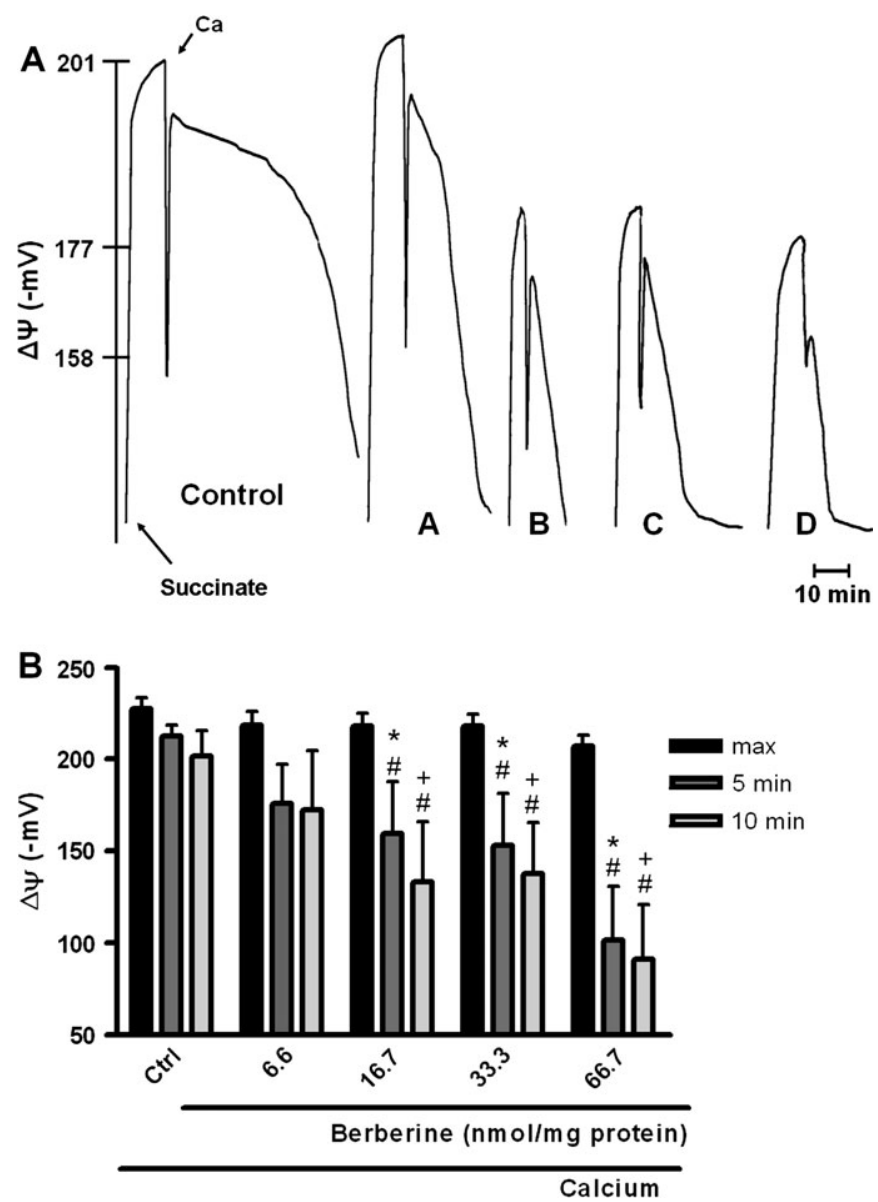

FIG. 5. Berberine stimulates calcium-induced depolarization. Freshly isolated mitochondria were energized with $5 \mathrm{mM}$ succinate/rotenone and different concentrations of berberine (berb) were added to the solution. Calcium (40-53.3 $\mathrm{nmol}$ of calcium $/ \mathrm{mg}$ protein) was added to initiate timedependent transmembrane potential alterations, measured by a $\mathrm{TPP}^{+}$electrode (A) Mitochondrial traces are representative of 7 repetitions, each using mitochondrial preparations from separate animals. The values corresponde to the $\Delta \psi$ before (max) and 5 or $10 \mathrm{~min}$ after calcium addition. $\mathrm{A}, 6.6 \mathrm{nmol}$ berb/ $\mathrm{mg}$ protein; $\mathrm{B}, 16.7 \mathrm{nmol}$ berb/mg protein; $\mathrm{C}, 33.3 \mathrm{nmol}$ berb/mg protein; $\mathrm{D}$, $66.7 \mathrm{nmol}$ berb/mg protein. (B) Bars represent the mean \pm SEM of seven repetitions each analyzed by one-way ANOVA. \#, max versus $5,10 \mathrm{~min}$ $(0,6.7,13.3,33.3$, and $66.7 \mathrm{nmol}$ berb/mg protein); *, control (Ctrl) $(5 \mathrm{~min})$ versus $5 \mathrm{~min}(6.7,13.3,33.3$, and $66.7 \mathrm{nmol}$ berb/mg protein); +, Ctrl (10 min) versus $10 \mathrm{~min}(6.7,13.3,33.3$, and $66.7 \mathrm{nmol}$ berb/mg protein). calcium levels were followed by using the fluorescent probe Calcium Green 5N. After an increase in probe fluorescence due to calcium addition, fluorescence decreased in all conditions tested, indicating mitochondrial calcium uptake. However, when berberine was present, calcium accumulation decreased (Figs. 7A and 7B) when compared with the control.

As in previous results, the most critical piece of data confirmed the berberine bypassed the inhibitory effect of bongkrekic acid on calcium accumulation, whereas Cyc A was able to prevent such effect. For the present study, only one berberine concentration $(133.3 \mathrm{nmol} / \mathrm{mg}$ protein) was used in order to investigate the effect of ANT modulators.

\section{Berberine does not Affect the Inhibitory Effect of ADP- Oligomycin on ANT}

Because bongkrekic acid did not inhibit the stimulatory effect of berberine on the MPT, a possibility is that the ANT would not be involved in the induction of the phenomenon by berberine. To demonstrate the opposite, the next set of experiments involved the use of ADP plus oligomycin, which also acts on the ANT to inhibit pore opening. In fact, ADP is an ANT ligand and a powerful MPT pore inhibitor (Crompton, 1999; Hagen et al., 2003). Oligomycin was added to avoid mitochondrial ADP conversion into ATP.

In the presence of calcium, 33.3 and $66.7 \mathrm{nmol}$ berberine $/ \mathrm{mg}$ protein decreases membrane potential in a concentration and time-dependent manner, as it was described before. When ADP-oligomycin was added to the mitochondrial suspension no berberine-induced calcium-induced $\Delta \Psi$ depolarization

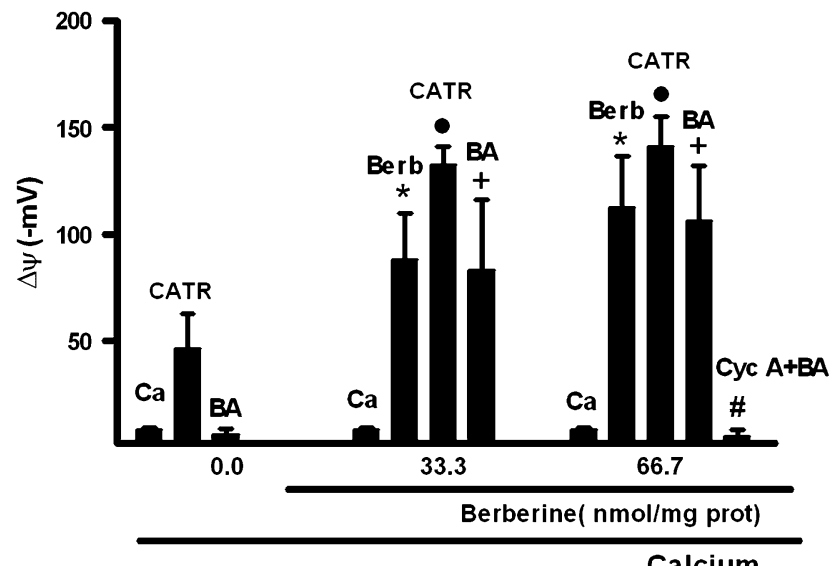

FIG. 6. Berberine decreases mitochondrial calcium accumulation independent of bongkrekic acid presence. Freshly isolated mitochondria were energized with $5 \mathrm{mM}$ succinate/rotenone and two different concentrations (33.3 and $66.7 \mathrm{nmol}$ berberine/mg protein) were added to the solution in the presence and absence of ANT inhibitors. Berberine induced a decrease in $\Delta \Psi$ time and concentration-dependent. Bars represent the mean \pm SEM of six separate experiments. - carboxyatractyloside (CATR) (0.00) versus CATR (33.33)/ CATR (66.67); *, calcium (Ca) versus Berberine (berb) (33.3/66.7); +, Bongkrekic acid (BA) versus BA (33.3)/BA (66.7); \#, BA (66.7) versus Cyc $\mathrm{A}+\mathrm{BA}(66.7)$, numbers represent berberine concentration. 

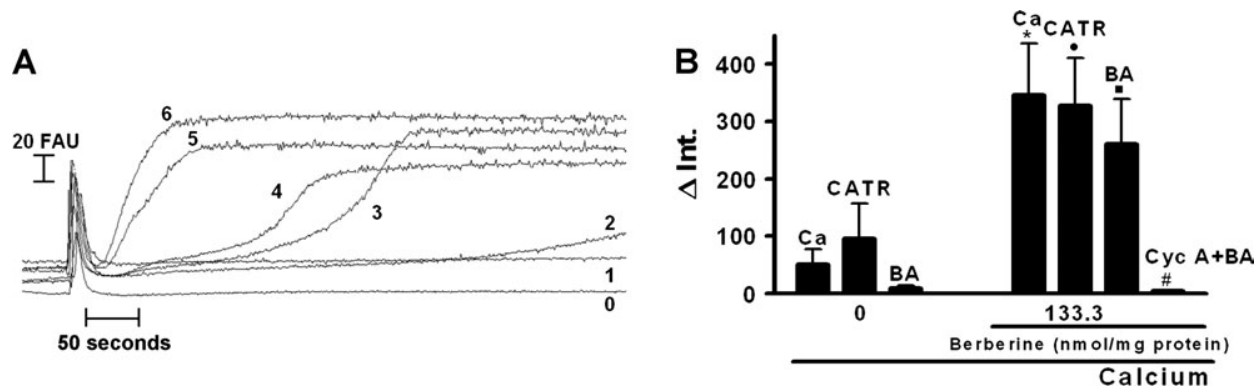

FIG. 7. Assessment of mitochondrial calcium loading capacity in presence of different concentrations of berberine and ANT inhibitors. The fluorescent probe Calcium Green 5-N $(1 \mu \mathrm{M})$ was used to evaluate the levels of extramitochondrial calcium as described under "Materials and Methods." (A) Mitochondrial traces are representative of six to seven repetitions, each using mitochondrial preparations from separate animals. (A) 0 —control (Ctrl); 1 -66.7 nmol berberine (berb)/ $\mathrm{mg}$ of protein; 2-133.3 nmol berb/mg of protein; $266.6 \mathrm{nmol}$ berb/mg of protein. (B) 0-Cyc A/berb/bongkrekic acid (BA); 1-BA; 2-Ctrl; 3-carboxyatractyloside (CATR); 4-BA + berb; 5-berb; 6-CATR + berb. (B) Values indicate the difference in fluorescence values before and 300 s after calcium (10-13.3 nmol/mg protein) addition. Bars represent the mean \pm SEM of six to seven repetitions. *, calcium (Ca) (0) versus Ca (133.3 berb); $\bullet$, CATR (0) versus CATR (133.3 berb); $\mathbf{\square}$, BA (0) versus BA (133.3 berb); \#, BA (133.3 berb) versus Cyc A + BA (133.3 berb).

occurred (Figs. 8A and $8 \mathrm{~B}$ ). In fact, $\Delta \Psi$ was maintained for several minutes after calcium addition.

\section{Berberine Affects the Depolarizing Effect of Oleic Acid on Mitochondria}

Oleic acid-induced uncoupling of mitochondria is known to be mediated by some anion carriers, including the ANT, as also by uncoupling proteins (UCPs) (Khailova et al., 2006).

We observe from Figure 9A that to each oleic acid addition, a small decrease in $\Delta \Psi$ occurs. We quantified the average $\Delta \Psi$ decay in the first four additions of oleic acid.

The data (Fig. 9B) indicate that the two of the berberine concentrations used increase the magnitude of oleic acid effects on $\Delta \Psi$. Surprisingly, carboxyatractyloside was not able to inhibit the depolarizing effect of oleic acid.

In order to verify if the effect of berberine on oleic acidinduced depolarization was due to the depressive effect on $\Delta \Psi$ (Fig. 9, and see also Pereira et al., 2007), we used a small amount of FCCP to clamp $\Delta \Psi$ to a value similar to the one obtained after incubation with $133.3 \mathrm{nmol}$ berberine/mg protein. No alteration on the effect of oleic acid was observed when the $\Delta \Psi$ was decreased with FCCP.

\section{The Initial Depolarization Effect of Berberine is not \\ Dependent on the ANT}

One particular effect observed when berberine is added to energized mitochondria is a sudden mitochondrial depolarization followed by a partial recovery of $\Delta \Psi$ to a value similar to what is observed when berberine is preincubated with mitochondria (Fig. 10A). By using the same concentrations of bongkrekic acid and carboxyatractyloside used before we confirmed that the immediate effects of berberine on mitochondrial $\Delta \Psi$ were not related with the ANT. In opposition and as expected, ADP phosphorylation was completely inhibited by both carboxyatractyloside and bongkrekic acid.

\section{DISCUSSION}

Phytomedicine, which involves the use of natural products from traditional herbal medicines for medical and healthfortifying purposes, is gaining international popularity. However, little is known about the mechanisms of action of their active principles (Tsai and Tsai, 2004) although formulations are widely in over the counter medications.

Previously, it was demonstrated that berberine increases oxidative stress on isolated liver mitochondria and melanoma cells. Increased oxidative stress is attributed to the interaction of berberine at a site downstream of the rotenone binding site (Pereira et al., 2007).

The inhibition of oxygen consumption by berberine is more pronounced when respiratory substrates for complex I are used, as described before (Barreto et al., 2003; Pereira et al., 2007). Despite this, succinate-sustained respiration is also inhibited, suggesting a larger range of targets in the respiratory chain (Pereira et al., 2007). The effects on the ADP/O ratio, ADP depolarization and lag phase (Pereira et al., 2007) suggests that berberine interacts with the mitochondrial phosphorylative system, although no effects on the mitochondrial ATPase were found.

Because it was also observed that berberine induces the calcium-induced MPT (Pereira et al., 2007), it is proposed that berberine may also act on the ANT, a proposed component of the pore complex.

The aim of the present investigation was then to identify a common target responsible for both disruption of mitochondrial bioenergetics and the induction of the MPT. Our work hypothesis is that the ANT is a target for berberine. Several end-points for MPT induction were used, including calciuminduced mitochondrial swelling, calcium-induced calcium release and calcium-induced mitochondrial depolarization. The three end-points are considered classic and sensitive techniques to follow MPT induction induced by xenobiotics (Bernardi and Forte, 2007). 

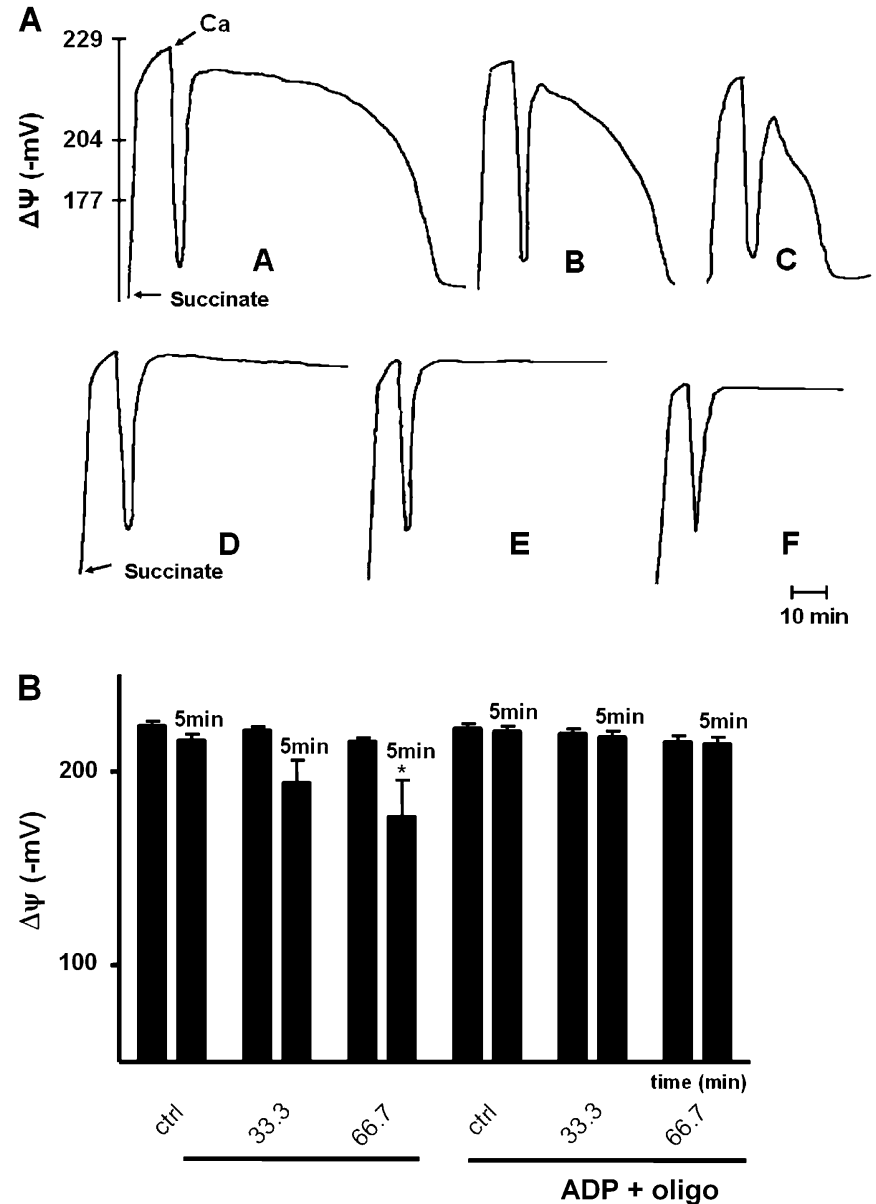

FIG. 8. ADP/oligomycin inhibits berberine stimulation of the MPT. A $\mathrm{TPP}^{+}$electrode was used to follow $\Delta \Psi$ variations that occur during the MPT. (A) Mitochondrial traces are representative of five different experiments. (A) A — control (Ctrl); B-33.3 nmol berberine (berb)/mg protein; C-66.7 nmol berb/mg protein; D-ADP + oligomycin (oligo); E-ADP + oligo + 33.3 $\mathrm{nmol}$ berb/mg protein; F-ADP + oligo $+66.7 \mathrm{nmol}$ berb/mg protein. (B) The data describe the value of $\Delta \Psi$ before and $5 \mathrm{~min}$ after calcium addition to isolated mitochondria. Bars represent the mean \pm SEM of five repetitions each analyzed by one-way ANOVA. (*) Statistically different compared with the control $(p<0.05)$. Legend: A-Ctrl; B-33.3 nmol berberine (berb)/mg protein; C-66.7 nmol berb/mg protein; D-ADP + oligo; E-ADP + oligo + $33.3 \mathrm{nmol}$ berb/mg protein; F-ADP + oligo $+66.7 \mathrm{nmol}$ berb/mg protein.

The results of this investigation demonstrate that berberine interacts with ANT on rat liver mitochondria, disturbing the effect of bongkrekic acid as a MPT pore inhibitor. Calciumdependent mitochondrial swelling (Fig. 3) induced by the test compound is in fact not inhibited by the ANT ligand bongkrekic acid. Also, in some of the experimental protocols used, berberine and carboxyatractyloside appear acting together to induce the MPT.

The results suggest a competition by berberine and bongkrekic acid for a common binding place, or a conformational change on the ANT or surrounding lipid membrane caused by berberine that decreases the affinity for bongkrekic acid.

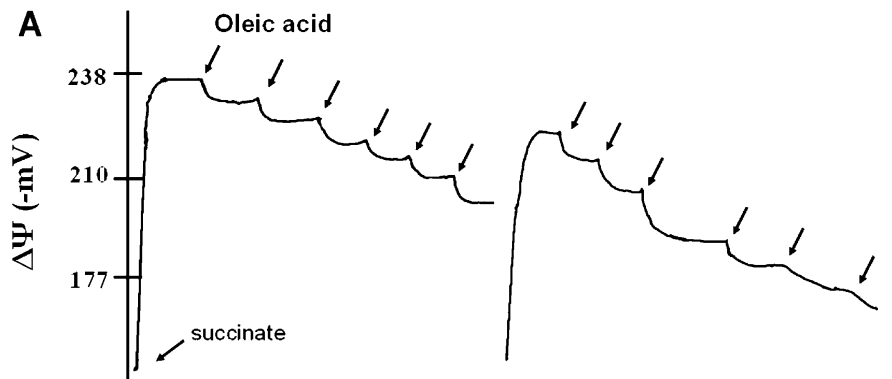

$66.7 \mathrm{nmol} / \mathrm{mg}$ prot. Berberine

$\stackrel{\longmapsto}{\text { minutes }}$

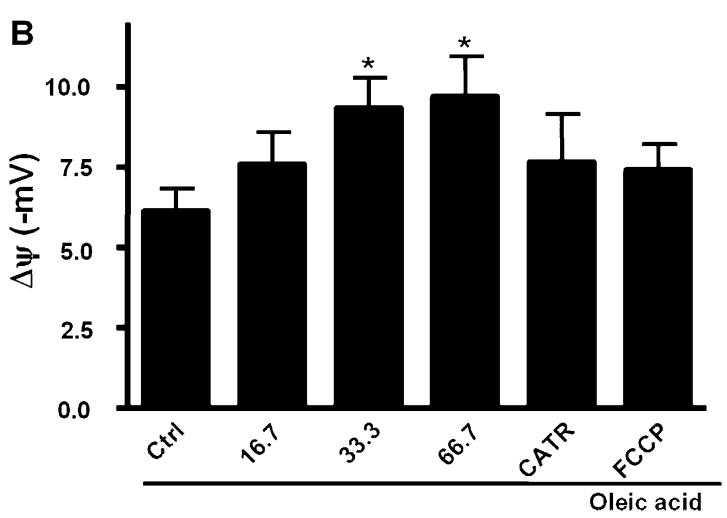

FIG. 9. Titration with oleic acid in the presence and absence of different concentrations of berberine. (A) Mitochondrial traces are representative of nine repetitions, each using mitochondrial preparations from separate animals. Each oleic acid addition was equivalent to $0.84 \mathrm{nmol}$ oleic acid/mg protein. (B) Berberine increased oleic acid-induced mitochondrial depolarization. The data indicate the average depolarization of four additions of oleic acid $=6.88 \mathrm{nmol}$ oleic acid/mg protein total. Bars represent the mean \pm SEM of nine repetitions. (*) Statistically difference compared with control.

Interestingly, berberine was not able to induce the permeability transition in nonenergized mitochondria which suggests that a polarized inner membrane is required for the effect of berberine, which is in accordance with previously published data indicating that berberine accumulation in mitochondria requires a transmembrane electrical potential.

Although berberine effects on the MPT were not inhibited by bongkrekic acid, ADP plus oligomycin completely prevented calcium-induced depolarization in the presence of berberine. Because ADP is a potent pore inhibitor due to its binding to the ANT (Crompton, 1999; Hagen et al., 2003), the result confirm that berberine stimulates the MPT through an interaction with the ANT and not to an unrelated mechanism.

Recently, it was reported that oleic acid stimulates mitochondrial uncoupling mediated by anionic transporters, such as the ANT, which causes a membrane potential decrease and an increase in the respiratory rate of mitochondria (Khailova et al., 2006). According to the fatty acid cycle hypothesis, UCP and the ANT participate in an uncoupling 

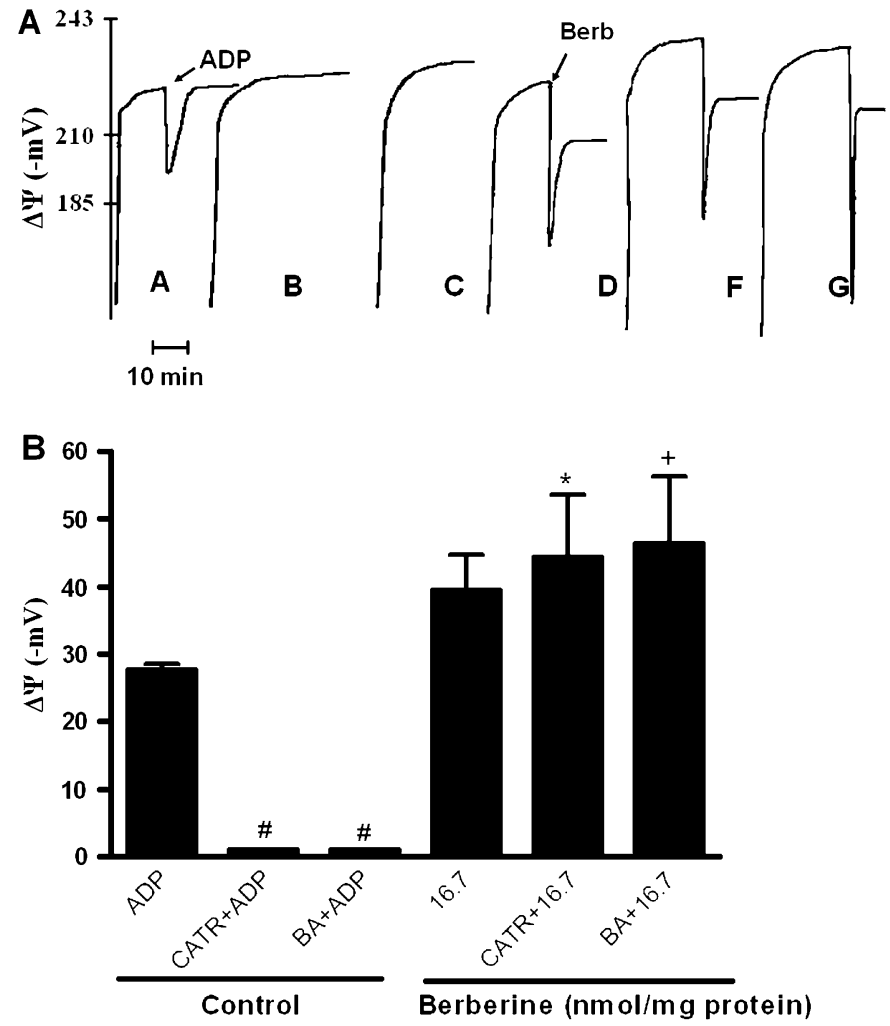

FIG. 10. Immediate depolarization of mitochondria by berberine is not inhibited by ANT inhibitors. (A) Mitochondrial traces are representative of eight repetitions, each using mitochondrial preparations from separate animals. (A) A — control (Ctrl); B — carboxyatractyloside (CATR); C—bongkrekic acid (BA) + ADP; D-Ctrl; E-CATR; F-BA (+ $16.7 \mathrm{nmol}$ berb/mg protein. (B) Berberine induced a fast and immediate mitochondrial depolarization even in the presence of CATR and BA. In a control situation (no berberine present) CATR and BA prevented ADP depolarization of mitochondria, by inhibiting the ANT. Bars represent the mean \pm SEM of eight repetitions each analyzed by one-way ANOVA. Differences were considered significant if the $p$ value was lower than 0.05. *, CATR + berb versus ADP/CATR; + , BA + berb versus ADP/BA; \#, ADP versus ADP/CATR and ADP/BA.

process by facilitating the efflux of fatty acid anions through hydrophobic barrier of the inner mitochondrial membrane (Skulachev, 1991). According to this, a titration with oleic acid was performed, in order to understand if berberine could also affect with the depolarizing effect of oleic acid, by means of an interaction with the ANT.

The results showed that berberine increases the effect of oleic acid. Depolarization caused by oleic acid in mitochondria was enhanced when different concentrations of berberine were added to the suspension. The effects of berberine on oleic acidinduced $\Delta \Psi$ depolarization were not due to the decrease in $\Delta \Psi$ caused by berberine alone, as a small amount of FCCP did not have the same effect. Interestingly and not as expected, carboxyatractyloside did not inhibit the action of berberine. The lack of inhibition is surprising after the results by Khailova et al. (2006) and O'Brien et al. (2008). Nevertheless, it is also likely that oleic acid effects are also different according to the conformational state of ANT. According to our data, apparently berberine facilitates the shuttling of oleic acid across the mitochondrial membrane.

Finally, the role of the ANT in the sudden depolarization observed upon berberine addition to a mitochondrial suspension was investigated. It must be stressed that the depolarization is transient and $\Delta \Psi$ partly recovers, although to a value lower than the control (Pereira et al., 2007).

The results show that berberine-induced depolarization was not related with the ANT, once carboxyatractyloside and bongkrekic acid had no effect (Fig. 10). The observation indicates that for the concentrations at which both ANT inhibitors hinder ADP phosphorylation, berberine-induced depolarization was not avoided. This depolarization is not Cyc A sensitive (data not shown) hence it is unlikely to be MPT related. The $\Delta \Psi$ depolarization is clearly different from the one occurring when berberine and calcium are coincubated with isolated mitochondrial fraction. In this case, it is the MPT pore opening that triggers the loss of $\Delta \Psi$.

Interaction of berberine with the ANT may help explaining the decreased ATP/ADP ratio found in melanoma cells (Pereira et al., 2007) and the range of effects on isolated mitochondrial fractions, although it must be stressed out that other ANTindependent targets may exist, as uncoupled respiration was also inhibited by berberine (Pereira et al., 2007).

The antitumor effect of berberine (Letasiova et al., 2006a, b; Pereira et al., 2007) appears to possess a mitochondrial component. Inhibition of the ANT in tumor cells can be an advantage if the process results in cell death through induction of the MPT and if a proper targeting and accumulation of the molecule occurs. In fact, the positive moiety in the molecule may be important for accumulating higher doses of berberine in tumor cells. Accumulation of positively charged molecules into mitochondria is driven by the negatively charged mitochondrial matrix. Interestingly, it was previously demonstrated that tumor development and progression is directly linked to the magnitude of mitochondrial $\Delta \psi$ in cancer cells (Heerdt et al., $2005,2006)$. It is reasonable to think that differences in mitochondrial membrane potential between subpopulations of tumor cells or even between normal and tumor cells (Dairkee and Hackett, 1991; Davis et al., 1985; Nadakavukaren et al., 1985; Ross et al., 2006) can be used to drive accumulation of positively charged antitumor molecules. As discussed earlier, berberine has other pharmacological indications although it is not clear at the moment if mitochondrial effects can account for its antimicrobial, antiarrhythmic or other pharmacological properties. Nevertheless, it has been described that inhibition of Complex I by berberine could account for its improvement of whole-body insulin sensitivity (Turner et al., 2008).

The data from the present work appear to show that berberine also presents some degree of toxicity to "nontumor" systems, which should be carefully understood. ANT inhibition in nontumor cells by berberine would be responsible for 
a decrease in energy production and could also result in MPT induction. To the best of our knowledge, no full toxicity assessment exists for berberine in humans, although its use in several commercially available supplements suggests that the compound may present a relatively wide safety interval. In fact, a study with patients with congestive heart failure treated with $1.2 \mathrm{~g} /$ day of oral berberine revealed low toxicity and resulted into an average plasma concentration of $0.11 \mathrm{mg} / \mathrm{l}$ which would translate into $0.3 \mu \mathrm{M}$ (Zeng and Zeng, 1999). Repeated cumulative treatments, alternative forms of formulation (e.g., topical application vs. injection) or more importantly, active mitochondrial accumulation due to its positive charge would be expected to increase its concentration in cells into the range of concentrations used in this study.

Empirical data from nontraditional medicines plus the use of extensive clinical assays would allow the use of berberine as a promising antimelanoma agent while maintaining its safety for humans. In radial/vertical forms of melanoma, a possible topical application of berberine would also be possible, thus minimizing side effects on other organs.

In conclusion, the present work identifies the ANT as an important target for berberine, with clear relevance for its proposed antitumor effects.

\section{REFERENCES}

Armstrong, J. S. (2006). The role of the mitochondrial permeability transition in cell death. Mitochondrion 6, 225-234.

Asai, M., Iwata, N., Yoshikawa, A., Aizaki, Y., Ishiura, S., Saido, T. C., and Maruyama, K. (2007). Berberine alters the processing of Alzheimer's amyloid precursor protein to decrease Abeta secretion. Biochem. Biophys. Res. Commun. 352, 498-502.

Barreto, M. C., Pinto, R. E., Arrabaca, J. D., and Pavao, M. L. (2003). Inhibition of mouse liver respiration by Chelidonium majus isoquinoline alkaloids. Toxicol. Lett. 146, 37-47.

Bernardi, P., and Forte, M. (2007). The mitochondrial permeability transition pore. Novartis Found. Symp. 287, 157-164; discussion 164-169.

Broekemeier, K. M., Dempsey, M. E., and Pfeiffer, D. R. (1989). Cyclosporin $\mathrm{A}$ is a potent inhibitor of the inner membrane permeability transition in liver mitochondria. J. Biol. Chem. 264, 7826-7830.

Ckless, K., Schlottfeldt, J. L., Pasqual, M., Moyna, P., Henriques, J. A., and Wajner, M. (1995). Inhibition of in-vitro lymphocyte transformation by the isoquinoline alkaloid berberine. J. Pharm. Pharmacol. 47, 1029-1031.

Crompton, M. (1999). The mitochondrial permeability transition pore and its role in cell death. Biochem. J. 341(Pt 2), 233-249.

Crompton, M., Virji, S., and Ward, J. M. (1998). Cyclophilin-D binds strongly to complexes of the voltage-dependent anion channel and the adenine nucleotide translocase to form the permeability transition pore. Eur. $J$. Biochem. 258, 729-735.

Dairkee, S. H., and Hackett, A. J. (1991). Differential retention of rhodamine 123 by breast carcinoma and normal human mammary tissue. Breast Cancer Res. Treat. 18, 57-61.

Davis, S., Weiss, M. J., Wong, J. R., Lampidis, T. J., and Chen, L. B. (1985). Mitochondrial and plasma membrane potentials cause unusual accumulation and retention of rhodamine 123 by human breast adenocarcinoma-derived MCF-7 cells. J. Biol. Chem. 260, 13844-13850.
Gornall, A. G., Bardawill, C. J., and David, M. M. (1949). Determination of serum proteins by means of the biuret reaction. J. Biol. Chem. 177, 751-766.

Hagen, T., Lagace, C. J., Modica-Napolitano, J. S., and Aprille, J. R. (2003). Permeability transition in rat liver mitochondria is modulated by the ATPMg/Pi carrier. Am. J. Physiol. Gastrointest. Liver Physiol. 285, G274-G281.

Haworth, R. A., and Hunter, D. R. (2000). Control of the mitochondrial permeability transition pore by high-affinity ADP binding at the ADP/ATP translocase in permeabilized mitochondria. J. Bioenerg. Biomembr. 32, 91-96.

Heerdt, B. G., Houston, M. A., and Augenlicht, L. H. (2005). The intrinsic mitochondrial membrane potential of colonic carcinoma cells is linked to the probability of tumor progression. Cancer Res. 65, 9861-9867.

Heerdt, B. G., Houston, M. A., and Augenlicht, L. H. (2006). Growth properties of colonic tumor cells are a function of the intrinsic mitochondrial membrane potential. Cancer Res. 66, 1591-1596.

Hong, Y., Hui, S. C., Chan, T. Y., and Hou, J. Y. (2002). Effect of berberine on regression of pressure-overload induced cardiac hypertrophy in rats. Am. $J$ Chin. Med. 30, 589-599.

Hunter, D. R., and Haworth, R. A. (1979). The Ca2+-induced membrane transition in mitochondria. I. The protective mechanisms. Arch. Biochem. Biophys. 195, 453-459.

Juhaszova, M., Wang, S., Zorov, D. B., Nuss, H. B., Gleichmann, M., Mattson, M. P., and Sollott, S. J. (2008). The identity and regulation of the mitochondrial permeability transition pore: Where the known meets the unknown. Ann. N. Y. Acad. Sci. 1123, 197-212.

Kamo, N., Muratsugu, M., Hongoh, R., and Kobatake, Y. (1979). Membrane potential of mitochondria measured with an electrode sensitive to tetraphenyl phosphonium and relationship between proton electrochemical potential and phosphorylation potential in steady state. J. Membr. Biol. 49, 105-121.

Kaneda, Y., Torii, M., Tanaka, T., and Aikawa, M. (1991). In vitro effects of berberine sulphate on the growth and structure of Entamoeba histolytica, Giardia lamblia and Trichomonas vaginalis. Ann. Trop. Med. Parasitol. 85, 417-425.

Khailova, L. S., Prikhodko, E. A., Dedukhova, V. I., Mokhova, E. N., Popov, V. N., and Skulachev, V. P. (2006). Participation of ATP/ADP antiporter in oleate- and oleate hydroperoxide-induced uncoupling suppressed by GDP and carboxyatractylate. Biochim. Biophys. Acta 1757, 1324-1329.

Letasiova, S., Jantova, S., Cipak, L., and Muckova, M. (2006a). Berberineantiproliferative activity in vitro and induction of apoptosis/necrosis of the U937 and B16 cells. Cancer Lett. 239, 254-262.

Letasiova, S., Jantova, S., Miko, M., Ovadekova, R., and Horvathova, M. (2006b). Effect of berberine on proliferation, biosynthesis of macromolecules, cell cycle and induction of intercalation with DNA, dsDNA damage and apoptosis in Ehrlich ascites carcinoma cells. J. Pharm. Pharmacol. 58, 263-270.

Letasiova, S., Jantova, S., Muckova, M., and Theiszova, M. (2005). Antiproliferative activity of berberine in vitro and in vivo. Biomed. Pap. Med. Fac. Univ. Palacky Olomouc Czech Repub. 149, 461-463.

Lin, S., Tsai, S. C., Lee, C. C., Wang, B. W., Liou, J. Y., and Shyu, K. G. (2004). Berberine inhibits HIF-1alpha expression via enhanced proteolysis. Mol. Pharmacol. 66, 612-619.

Nadakavukaren, K. K., Nadakavukaren, J. J., and Chen, L. B. (1985). Increased rhodamine 123 uptake by carcinoma cells. Cancer Res. 45, 6093-6099.

O'Brien, T. M., Oliveira, P. J., and Wallace, K. B. (2008). Inhibition of the adenine nucleotide translocator by $\mathrm{N}$-acetyl perfluorooctane sulfonamides in vitro. Toxicol. Appl. Pharmacol. 227, 184-195.

Pereira, G. C., Branco, A. F., Matos, J. A., Pereira, S. L., Parke, D., Perkins, E. L., Serafim, T. L., Sardao, V. A., Santos, M. S., Moreno, A. J., et al. (2007). Mitochondrially targeted effects of berberine [Natural Yellow 18, 5,6-dihydro-9,10-dimethoxybenzo(g)-1,3-benzodioxolo(5,6-a) 
quinolizinium] on K1735-M2 mouse melanoma cells: Comparison with direct effects on isolated mitochondrial fractions. J. Pharmacol. Exp. Ther. 323, 636-649.

Ross, M. F., Da Ros, T., Blaikie, F. H., Prime, T. A., Porteous, C. M., SeverinaSkulachev, V. P., II., Kjaergaard, H. G., Smith, R. A., and Murphy, M. P. (2006). Accumulation of lipophilic dications by mitochondria and cells. Biochem. J. 400, 199-208.

Serafim, T. L., Oliveira, P. J., Sardao, V. A., Perkins, E., Parke, D., and Holy, J. (2008). Different concentrations of berberine result in distinct cellular localization patterns and cell cycle effects in a melanoma cell line. Cancer Chemother. Pharmacol. 61, 1007-1018.

Shirwaikar, A., Rajendran, K., and Punitha, I. S. (2006). In vitro antioxidant studies on the benzyl tetra isoquinoline alkaloid berberine. Biol. Pharm. Bull. 29, 1906-1910.

Skulachev, V. P. (1991). Fatty acid circuit as a physiological mechanism of uncoupling of oxidative phosphorylation. FEBS Lett. 294, 158-162.

Szabo, I., and Zoratti, M. (1991). The giant channel of the inner mitochondrial membrane is inhibited by cyclosporin A. J. Biol. Chem. 266, 3376-3379.
Taylor, C. T., and Baird, A. W. (1995). Berberine inhibition of electrogenic ion transport in rat colon. Br. J. Pharmacol. 116, 2667-2672.

Tsai, P. L., and Tsai, T. H. (2004). Hepatobiliary excretion of berberine. Drug Metab. Dispos. 32, 405-412.

Turner, N., Li, J. Y., Gosby, A., To, S. W., Cheng, Z., Miyoshi, H., Taketo, M. M., Cooney, G. J., Kraegen, E. W., James, D. E., et al. (2008). Berberine and its more biologically available derivative, dihydroberberine, inhibit mitochondrial respiratory complex I: A mechanism for the action of berberine to activate AMP-activated protein kinase and improve insulin action. Diabetes 57, 1414-1418.

Vergun, O., and Reynolds, I. J. (2005). Distinct characteristics of $\mathrm{Ca}(2+)$ induced depolarization of isolated brain and liver mitochondria. Biochim. Biophys. Acta 1709, 127-137.

Zeng, X., and Zeng, X. (1999). Relationship between the clinical effects of berberine on severe congestive heart failure and its concentration in plasma studied by HPLC. Biomed. Chromatogr. 13, 442-444.

Zhou, L., Yang, Y., Wang, X., Liu, S., Shang, W., Yuan, G., Li, F., Tang, J., Chen, M., and Chen, J. (2007). Berberine stimulates glucose transport through a mechanism distinct from insulin. Metabolism 56, 405-412. 